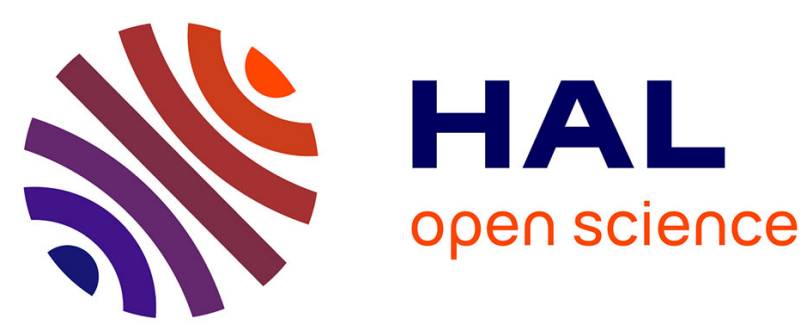

\title{
Propositions About Novelty
}

Ulrich Witt

\section{- To cite this version:}

Ulrich Witt. Propositions About Novelty. Journal of Economic Behavior and Organization, 2009, 70

(1-2), pp.311. 10.1016/j.jebo.2009.01.008 . hal-00688183

\section{HAL Id: hal-00688183 \\ https://hal.science/hal-00688183}

Submitted on 17 Apr 2012

HAL is a multi-disciplinary open access archive for the deposit and dissemination of scientific research documents, whether they are published or not. The documents may come from teaching and research institutions in France or abroad, or from public or private research centers.
L'archive ouverte pluridisciplinaire HAL, est destinée au dépôt et à la diffusion de documents scientifiques de niveau recherche, publiés ou non, émanant des établissements d'enseignement et de recherche français ou étrangers, des laboratoires publics ou privés. 


\section{Accepted Manuscript}

Title: Propositions About Novelty

Authors: Ulrich Witt

PII: $\quad$ S0167-2681(09)00027-4

DOI: $\quad$ doi:10.1016/j.jebo.2009.01.008

Reference: $\quad$ JEBO 2307

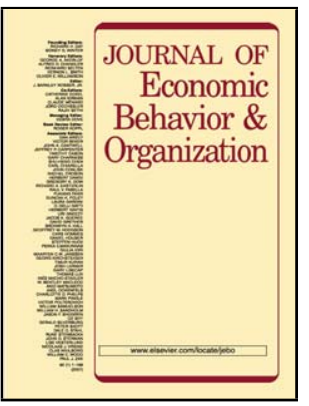

To appear in: Journal of Economic Behavior \& Organization

Received date: $\quad 15-6-2007$

Revised date: $\quad 19-1-2009$

Accepted date: $\quad$ 19-1-2009

Please cite this article as: Witt, U., Propositions About Novelty, Journal of Economic Behavior and Organization (2008), doi:10.1016/j.jebo.2009.01.008

This is a PDF file of an unedited manuscript that has been accepted for publication. As a service to our customers we are providing this early version of the manuscript. The manuscript will undergo copyediting, typesetting, and review of the resulting proof before it is published in its final form. Please note that during the production process errors may be discovered which could affect the content, and all legal disclaimers that apply to the journal pertain. 


\title{
Propositions About Novelty
}

\author{
by Ulrich Witt*) \\ Max Planck Institute of Economics \\ Jena, Germany \\ Ulrich.Witt@econ.mpg.de
}

\begin{abstract}
The emergence of novelty is a ubiquitous feature in science, technology, and economic life. It is the crucial input to the growth of human knowledge. At the same time, novelty is one of the most amorphous concepts in scientific thought. Theorizing about novelty and its emergence faces notorious problems. This paper explores why this is so and what can be done to deal in a more systematic fashion with novelty. The notion of degrees of novelty is introduced, and its relationship with the concept of uncertainty is discussed. The results of the inquiry are summarized by a number of hypotheses.
\end{abstract}

Key words: novelty, creativity, evolution, uncertainty, probability, knowledge growth

JEL classification: $\quad$ D 80, D 83, O 30, O 31

*) The author is grateful to the editor and an anonymous referee of this journal for helpful comments. Thanks also to the participants of the American Institute for Economic Research Conference on Cognition and Economics, Great Barrington MA. 2003, the KurtDopfer-Seminar of the University of St. Gall 2005, and the Conference on Innovation and Complexity, Pollenzo, Italy 2007 commenting on earlier drafts of this paper. The usual disclaimer applies. 


\section{Introduction}

The emergence of novelty is a pervasive feature of modern life and an important driver of economic development. New ideas, practices, and artefacts transform culture, commerce, and technology. When considering options to innovate, every businessman is contemplating, and often trying out, new speculations, beliefs, and know-how. More broadly seen, in nature too, the emergence of genetic and epigenetic novelty is a driving agent of evolution. Both in nature and the sphere of human action, novelty adds to already existing variety and fuels the competitive transformation processes with manifest consequences. In the economy, the continuing creation of novelty feeds the growth of useful (problem-solving) knowledge that, in turn, has been the mainstay of the soaring economic growth of the past few centuries.

Despite the importance of emerging novelty as an agent of change, a comprehensive, structured theory of novelty does not exist, and it does not even seem clear whether such a theory is feasible at all. The complexity of the act from which novelty emerges and its epistemic peculiarity (the fact that the informational content of novelty cannot be anticipated) make novelty a difficult research topic. Some insights that are relevant for the mental production of novelty can be found in the vast literature on human creativity (see, e.g., Sternberg 1999), cognitive science (e.g., in the work on problem solving, see Newell and Simon 1972), and the formation of cognitive concepts (Finke et al. 1992, Hofstadter et al. 1995, Fauconnier and Turner 2002). There have even been some first attempts at dealing with novelty analytically (e.g., in Zabell 1992). In contrast, with the exception of historical studies of the emergence of man-made novelty in technology and production (e.g., Usher 1954, Vincenti 1990, Mokyr 1990), in economics the emergence of novelty is usually treated as an exogenous shock. If novelty is considered at all, the focus is on its diffusion in an industry or the economy.

In this paper, the emergence of novelty will therefore be put at center stage. What the 


\section{-3-}

processes that lead to novelty emergence look like and what kind of hypotheses are possible ex ante about their outcome (i.e. about the emerging informational content) will be explored. The paper proceeds as follows. Section II clarifies some conceptual ambiguities that often hamper the discussion about novelty. Section III introduces a procedural approach to novelty that distinguishes between a generative operation and an interpretative operation that are involved in the emergence of novelty. These two operations are shown to be logically independent of each other and to have profoundly differing implications. In the light of the procedural approach suggested, Section IV discusses the epistemic peculiarities in the creation of novelty, the logical structure of the interpretative operation, and the conclusions to be drawn regarding the role that novelty plays for the evolution of human knowledge. Section V elaborates on the observation that, in retrospect, it makes a difference whether novelty means only a minor variation in known details or an entirely new thought that eludes existing frameworks. The notion of degrees of novelty is introduced and is compared with the concepts of degrees of likelihood or belief. Section VI offers the conclusions.

\section{Some Prolegomena}

When an attempt is made in this paper to explore the possibilities for and some features of a theory of novelty and its emergence, one problem that must be faced is the ambiguity of the term "novelty". Usually, what is meant can be captured as follows:

Definition Novelty is something that was unknown before a particular point in time and that, hence, was discovered or created at that point in time.

Some qualifications are, however, necessary. Novelty is defined here relative to existing propositional or procedural knowledge on facts, rules, or theories. This relationship implies several contingencies. What is not known in one domain $\mathrm{j}$ up to a time $\mathrm{t}_{\mathrm{j}}$ can already have become known in a different 
domain $i$ at a time $t_{i}<t_{j}$. It is thus new only to the specific domain $j$. Therefore, it is necessary to distinguish between something novel to a specific domain and something that is universally novel. Universal novelty emerges somewhere at a time $\tau$, so that there is no domain $\mathrm{i}$ for which $\mathrm{t}_{\mathrm{i}}<\tau$.

The distinctive epistemological feature of novelty is expressed by the difference it makes when the ex ante view of what is novel (i.e. the view at a time $\mathrm{t}<\tau$ ) is compared to the view one can take ex post (i.e. at time $\mathrm{t} \geq \tau$ ). Unlike in the ex ante case, ex post a (fictitious) observer is able, and typically is assumed, to know the relevant properties and meaning of what has emerged or is being created. However, what has become known in this way is no longer "novelty" in the epistemic sense. It is an ordinary piece of information labeled "novel" only in a retrospective sense. Depending on which perspective is taken, theorizing about novelty thus amounts to either pre-revelation or postrevelation analysis, two epistemologically fundamentally different approaches (Witt 1993). One of the consequences of the epistemic limitations that are binding up to time $\tau$ is the predictive weakness of scientific disciplines in whose domains novelty is regularly generated in the course of events, economics being a case in point.

As far as anthropogenic novelty is concerned, its origin can be exogenously triggered new sensory perceptions (and their mental representation) or the endogenous formation of new thought. Discovering, for example, the flavor of a not-yet-tasted fruit certainly satisfies the above definition of novelty. However, this may, but does not have to, involve a creative act of the mind, something that, in contrast, is always the case when new thought is formed. This distinction is relevant for clarifying the relationship between discovery and invention. Discovery can, but does not necessarily, involve a creative act. Invention, in contrast, always presupposes the creation of some new thought that materializes in the form of new artefact and/or practice. On the one side there are thus some discoveries that do not involve a creative act while, on the other side, there are both discoveries and inventions that do.

New ideas, beliefs, and know-how induce the agents to develop new behaviors, strategies, 
$-5-$

practices, and artefacts ("innovations"). The differences between domain-specific and universal novelty turns up here again in the guise of subjective vs. objective novelty (Machlup 1980, chap. 8, Witt 1993, in Boden 1999 as historic vs. psychological novelty). Subjective novelty is something that is new for a particular agent $\mathrm{k}$ who gets to know it at time $\mathrm{t}_{\mathrm{k}}$. Being new relative to k's knowledge domain does not exclude the possibility that its meaning is already known to some other agent(s),for example, a scientific observer $h$, if $\tau \leq t_{h}<t_{k}$. Already knowing what is going to be revealed to other individuals later provides a basis for conjecturing about their likely reactions when they become aware of the particular novelty. This is indeed the epistemic position regularly adopted in research on the diffusion of innovations (see Rogers 1995), the arena of post-revelation analysis. In the case of objective, that is, universal, novelty (the arena of pre-revelation analysis), this epistemic position is not possible.

\section{The Procedural Approach: Operations by Which Novelty Is Being Created}

What can be said more precisely about how new ideas, beliefs, and knowledge emerge, given that, as Shackle (1973, p. 26) noted, such statements cannot be logically deduced from already existing ideas, beliefs, and knowledge? What is the logical relationship between already existing thought and new thought established by the creative act? In his inquiry into the emergence of cognitive novelty, Koestler (1964, p. 120) claims that "the creative act ... does not create something out of nothing; it uncovers, selects, re-shuffles, combines, synthesizes already existing facts, ideas, faculties, skills”. By a more or less accidental displacement of attention to something not previously noted, new elements are introduced into a given context with which they have not been previously associated.

The idea that novelty can emerge from cross-over or, more generally, (re-) combination of existing elements (including combination with omissions), is not a new one, but it needs some qualifications. An arbitrary (re-) combination of something with something else is rarely what leads to 
$-6-$

new ideas or imaginings. ${ }^{1}$ A cognitive concept carries meaning only in relation to some context. To combine concepts in an accidental way therefore usually means mixing up contexts that are foreign to one another. New meaning can only be induced if there is a chance of aligning the different contexts (Gentner and Markman 1997). However, even if the contextual constraint of some similarity between the things to be (re-) combined is met, the subsequent reshuffling does not by itself lend a new meaning to the resulting combinations. For this to be accomplished, a meaningful association between them must be induced, be it a "conceptual integration" based on the common elements or features or a categorical reduction by which commonalities are induced via a memorized more general category (Finke et al. 1992, Chap. 2 and 5, Fauconnier and Turner 2002, Chap.3).

1 Jonathan Swift (1934, p. 172, reprint of $1^{\text {st }}$ edition of 1726) parodied the absurdity of such a notion nicely in Section III of Gulliver's Travels, "A Voyage to Laputa," with a mechanical reshuffling of words. The protagonist Gulliver visits the academy of science, meeting the "projector of speculative learning" who shows him

“.... a Frame, about the Sides whereof all his Pupils stood in Ranks. It was Twenty Foot square. The Superficies was composed of several Bits of Wood, about the Bigness of a Dye, but some larger than others. They were all linked together by slender Wires. These Bits of Wood were covered on every Square with Papers pasted on them; and on these Papers were written all the Words of their Language ...without any Order. The Professor then desired me to observe, for he was going to set his Engine at work. The Pupils at his Command took each of them hold of an Iron Handle, whereof there were Forty fixed round the Edges of the Frame; and giving them a sudden Turn, the whole Disposition of Words was entirely changed. He then commanded Six and Thirty of the Lads to read the several Lines softly as they appeared upon the frame; and where they found three or four Words together that might make Part of the Sentence, they dictated to the four remaining Boys..." (Swift 1934 , p. 172 , reprint of $1^{\text {st }}$ edition of 1726 ) 
$-7-$

At a closer look it thus appears that a qualified reshuffling of existing elements is one thing. Finding sense in the resulting combinations, if there is any, for example, aligning the multiple contexts that have been mingled, is something else. From a procedural point of view on what happens when novelty emerges, one may thus conclude

Hypothesis 1 The creation of new cognitive concepts (ideas, imaginings) involves two operations. One is a generative operation that produces new (re-) combinations of elements. The other is an interpretative operation by which the new (re-) combination is integrated into a newly emerging or a more general already existing concept.

Even though they coincide in time, the two operations are logically distinct. How they work and interact can be illustrated by a simple example of a conceptual integration.

Economic activities can sometimes be described by simple factual statements such as, "agent X sells commodity Y”. In a semantic representation, the cognitive concepts involved are depicted by subject $\mathrm{S}$, predicate $\mathrm{P}$, and object $\mathrm{O}$ in the corresponding basic sentence. (Using a semantic representation has some advantages. First, the criteria constraining the generative operation can easily be identified. Second, at the semantic level, it is possible to grasp intuitively the similarity of cognitive concepts and the meaning that emerges in the act of conceptual integration.) Consider the following simple statements

(i-a) "kiosks $\left(\mathrm{S}_{1}\right)$ sell $\left(\mathrm{P}_{\mathrm{a}}\right)$ convenience goods $\left(\mathrm{O}_{1 a}\right)$ "

and

(i-b) "kiosks $\left(\mathrm{S}_{1}\right)$ have $\left(\mathrm{P}_{\mathrm{b}}\right)$ extended business hours $\left(\mathrm{O}_{\mathrm{b}}\right)$ ". 
$-8-$

Statements like these, sharing the same subject ("kiosks"), are simple propositional networks by which contextual knowledge or beliefs about the subject are encoded (Anderson 2000, chap. 5). Imagine a test person who is asked to write down what she associates with "kiosk" (i.e. retrieves from her memory as the relevant context). The results typically are statements such as (i-a) and (i-b). Let us denote the structure of the corresponding propositional network by

$$
\mathrm{P}_{\mathrm{a}} O_{1 a}
$$

$$
\mathrm{S}_{1}
$$
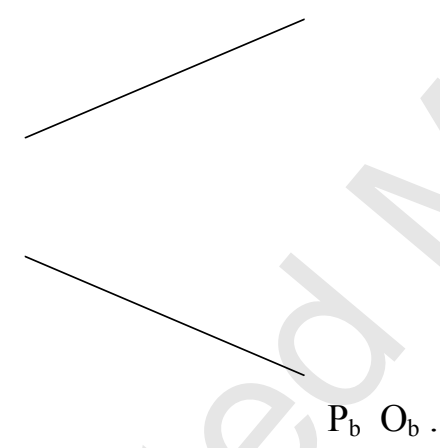

Now suppose that the test person is given the cognitive concept "gas station" $\left(\mathrm{S}_{2}\right)$, and that she associates the following statements:

(ii-a) "gas stations $\left(\mathrm{S}_{2}\right)$ sell $\left(\mathrm{P}_{\mathrm{a}}\right)$ gas $\left(\mathrm{O}_{2 a}\right) "$

and

(ii-b) "gas stations $\left(\mathrm{S}_{2}\right)$ have $\left(\mathrm{P}_{\mathrm{b}}\right)$ extended business hours $\left(\mathrm{O}_{\mathrm{b}}\right)$ ". 
$-9-$

The corresponding propositional network can be denoted by

$$
\mathrm{P}_{\mathrm{a}} O_{2 a}
$$

(2)

$\mathrm{S}_{2}$
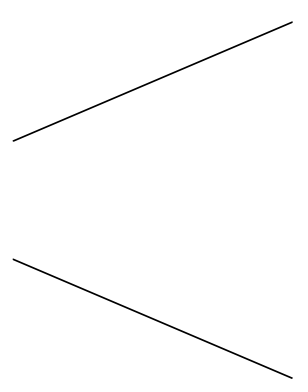

$\mathrm{P}_{\mathrm{b}} \mathrm{O}_{\mathrm{b}}$.

The propositional networks (1) and (2) have $\mathrm{P}_{\mathrm{a}}, \mathrm{P}_{\mathrm{b}}$, and $\mathrm{O}_{\mathrm{b}}$ in common. To avoid nonsensical statements that would result from an arbitrary reshuffling of subjects, predicates, and objects (as in Swift's parody), the commonalities can be used as a basis for cross-overs or recombinations. The generative operation is then subjected to the constraint of retaining the syntactic order. This means that, in the recombination, precisely the non-common elements (set in italics) are crossed over as shown by the scheme in (3).

$\mathrm{S}_{1}$

$\mathrm{P}_{\mathrm{a}} O_{1 a}$

(3)

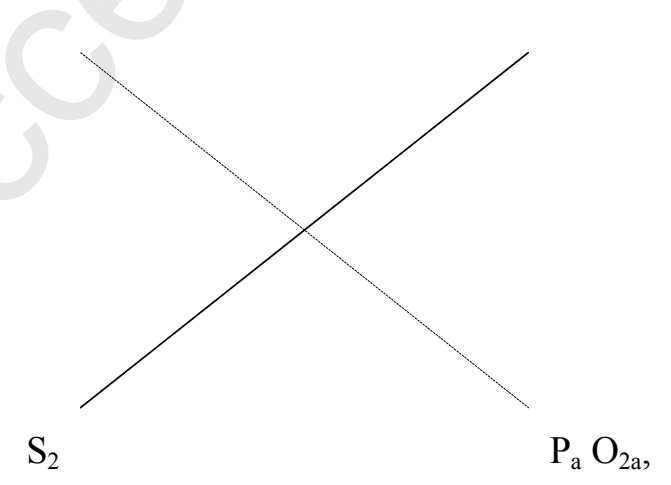


$-10-$

For the two statements thus generated, the question is whether, in the new combination, the original contexts can be aligned so that a new meaning is induced. This question is answered by the interpretative operation. In the present case, the original contexts are so similar that they suggest a combinatory extension of the original factual statements (i-a) and (ii-a) into

(i-c) "kiosks sell convenience goods and gas"

(ii-c) "gas stations sell gas and convenience goods". 2

The combinatory extensions (i-c) and (ii-c) evoke the interpretation of a possible extension of the business strategies of kiosks and gas stations respectively. Despite the extremely simple structure of the propositional networks used in the generative operation, when interpreted as a combinatory extension, its outcome yields meaningful new concepts. In the 1960s this extension was indeed an innovation strategy in the services industry. In the U.S., convenience store chains such as "7-eleven" started adding gas to their assortment of products and services. About a decade later, gas stations in Europe started to sell convenience goods in addition to gas to take advantage of their extended business hours.

2 An equivalent result can be derived if the statements (i-a) and (ii-a) are interpreted as definitions of the form "kiosks are businesses that sell convenience goods" and "gas stations are businesses that sell gas". A mere recombinant exchange of $\mathrm{O}_{1 \mathrm{a}}$ and $\mathrm{O}_{2 \mathrm{a}}$ would result in a violation of these definitions. Only by expanding the definitions of kiosks and gas stations in the form of the combinatory extension do the new, conceptually integrated definitions "kiosks are businesses that sell convenience goods and gas" and "gas stations are businesses that sell gas and convenience goods" emerge. 


\section{Some Implications of the Procedural Approach to Novelty}

The example in the previous section served to highlight the different functions of the generative and the interpretative operation. Due to the simple structure of the factual statements in the example, both the generative operation and the combinatory extensions identified by the interpretative operation are rather special. They can easily be extended to explain the use of metaphors and analogies by which the acts of recombining and conceptual integration in creating novelty are often systematized (see, e.g., the work on concept formation by Nersessian 2002). In other cases, the associative basis for attributing new meaning may, of course, require a more complex semantic representation and/or logic, such as, when meaning is induced by categorical reduction and generic abstraction. Furthermore, before a conceptual integration succeeds, several iterations may often be necessary in which the conceptual inputs to the generative operation are modified or replaced over and over again (Finke et al. 1992, Chap.2). In addition, if objects other than semantically represented ones are involved, the two operations may have to be suitably adjusted. However, these reservations notwithstanding, both the generative operation and the interpretative operation are generic to the creation of mental novelty. Their exemplary discussion can therefore be used to derive some more general insights about novelty and its emergence that will be discussed now.

A first inference that can be made relates to the generative operation. With reference to Campbell (1960), the adherents of an evolutionary epistemology often claim that novelty emerges from "blind" variation (see, e.g., Hodgson and Knudsen 2006). Blindness here means that the contextual constraints on the (re-) combination process discussed above are not binding. Any arbitrarily chosen sets such as, for example, the English vocabulary and the data set of daily DowJones stock exchange indices from 1950 - 2000 could be commingled irrespective of whether or not there is some commonality on the basis of which the disparate elements could be conceptually integrated. Furthermore, in choosing which elements of the sets are to be (re-) combined, blind variation would also mean ignoring the internal structure of the sets such as, for example, the 
syntactic structure of the propositional networks in scheme (3) above.

However, even in genetics, the model for evolutionary epistemology, variation is not blind (not even in the case of mutations which are, after all, local copying errors in DNA strings). In sexual reproduction, for instance, the lion's share of variation results from the recombination of parental genes. This is subject to strict matching rules for the nucleotide chains in the breakage and reunion of homologous chromosomes, a biochemical form of rigid contextuality. Even in the analogously modeled genetic algorithms, the crossing over is confined to elements or sections of binary coded strings, and it exchanges only equivalently formatted sections. The following may therefore be inferred:

Hypothesis 2 The generative operation is based on a contextual pre-selection of sets and elements of sets to be recombined that follows some similarity or conformity criterion.

Because of the similarity and conformity criteria underlying the choice of what is (re-) combined, the generative operation is a more or less strongly guided process and, hence, is far from a "blind" variation.

A further conclusion concerns the interpretative operation and a significant feature in which it differs from the generative operation. Unlike the generative operation with its plain logic, the associative act by which the human mind accomplishes a conceptual integration in the interpretative operation is not fully understood. ${ }^{3}$ In discussing examples of the associative act such as the one above,

3 To say nothing of the underlying neural processes (cf. Koch and Crick 2000, Edelman and Tononi 2000, Chap. 10). Johnson-Laird (2005) considers the associative act as something that is outside the scope of current psychological theories and their computer implementations. It has been conjectured, however, that the associative act is a search process in an extremely high dimensional 


\section{$-13-$}

the unresolved problem of how exactly the conceptual integration comes about is by-passed by letting our intuition doing the trick. In view of this it is important that, by Hypothesis 1 , the generative operation and the interpretative operation are logically distinct and, hence, can be carried out separately from one another. From this follows

Hypothesis 3 While the generative operation can be automatized mechanically or electronically outside the human mind, for example, by numerical algorithms and programs, the carrying out of the interpretative operation is bound to the medium of the human mind and can therefore not be automatized.

A remark is necessary here. The generative and the interpretative operations are given a constitutive role in the creation of mental novelty. This does not necessarily mean that these two operations are also sufficient for the creative act. Yet another operation can often be observed to accompany the interpretative operation, namely an evaluative one. However, where the interpretative operation answers the question of what it is that emerges, the evaluative operation is concerned with what the utility, advantage, benefit of this is. Even if coinciding with the interpretative operation, the act of evaluating the novel outcome is therefore logically distinct and, from the procedural point of view, not necessary for novelty to be created. On the other hand, where novelty creation is an iterated process, a concomitant evaluative operation can feed back on the generative operation. This happens if the criteria on which, according to Hypothesis 2, the pre-selection of sets and elements involved in the generative operation is made contingent on how the preceding outcomes of the interpretative

memory space (cf. Kohonen 1987). By searching the space, connections are traced to cognitive concepts that have been laid out by earlier experience. These concepts can be imagined to be sampled with a probability that depends on how closely they appear to be connected to the new concept under consideration, yet thus far it has not been possible to derive the measure of coherence that is required to determine the "closeness" of concepts. 


\section{$-14-$}

operation are evaluated. Though not itself involved in the creation of novelty, an evaluative operation can in this way influence what novelty emerges.

The fact that an evaluative operation is logically distinct from the interpretative operation makes it possible to carry this out outside the human mind and to automatize it. Recently, steps in that direction have indeed been taken by modeling the feedback of the evaluative operation on the generative operation by way of an analogy to natural selection in genetic or, more generally, evolution algorithms (see Holland 1992, Bäck 1996). In nature, the generative operation is the recombination of specifically selected genetic material. The equivalent to the interpretative operation is the epigenetic expression of the varied gene code in the development of the phenotype. The evaluation is indirectly produced at an extra-somatic level by the competition for reproductive success between the different phenotypes that develop from the population's gene pool. The systematic feedback between the adaptive value of the traits of the phenotypes (evaluation) and the selective admission of their genes to the recombination (generative operation) in the next generation is called natural selection.

Analogously, in genetic algorithms, elements or segments of binary strings are recombined in a computer-based generative operation. If not skipped altogether, the interpretative operation is degenerated into pre-specified mapping instructions written into the computer program. The resulting "new" variant is launched into a simulated environment where it is subjected to an evaluative operation that measures the performance relative to that of competing variants produced in the same way. If better performing variants are used more frequently or exclusively for recombination in a next round of the simulated generative operation, this can shape the kind of novelty that emerges in the simulation over time.

By recognizing the role of such complex numerically iterated dynamics systems in generating novelty, yet another conclusion can be drawn. Assume that the algorithm of the generative operation is known and that it does not produce a chaotic time series. In addition, let the interpretative and/or 


\section{$-15-$}

evaluative operations be pre-specified. If it is nonetheless not possible to deduce the implications logically from the premises, this is usually because of the complexity of the recursive relation between the generative, interpretative, and evaluative operations. Novelty in the sense of not previously known implications can then be discovered (rather than created) by the human experimenter through numerical simulations. Hence, the process of revealing novelty by simulating complex recursive programs and the creative act of the human mind have something in common. The commonality is a key to understanding the peculiar epistemic features of novelty.

Hypothesis 4 Novelty is revealed through inductive operations whose outcome, by definition, cannot be derived from the premises. Carrying out the inductive operations requires time, and thus prevents the meaning of novelty being instantaneously accessible.

The inductive nature of the simulations discussed is obvious. Notwithstanding the fact that the creation of novelty in the human mind is not fully understood, the inductive nature of the associative act underlying conceptual integration, categorical reduction, or generic abstraction seems equally obvious. Moreover, it has been argued that the interpretative operation is inaccessible to deductive reasoning on grounds of principle (see Markose 2004 and 2005 referring to Gödel's incompleteness theorem). The generative operation requires inductive methods whenever it is chaotic (which can even be the case for simple recombination dynamics; see Prigogine 1993) or too complex to be solved analytically. Note that the time constraint stated in Hypothesis 4 still holds even if someday it would be possible to automatize the interpretative operation in a non-trivial way outside the human mind.

A final inference that can be drawn from the approach taken here concerns the role that novelty plays for the evolution of human thought and knowledge. In the example in the previous section, the meaning of the elements (the cognitive concepts in the propositional networks) from which the combinatory extension emerged were all known beforehand. Moreover, it seems likely that the primitive concepts ("gas station", "kiosk") themselves emerged through conceptual integration 
from other, still more primitive cognitive concepts. It can therefore be conjectured that the emergence of novelty is not only a process that is iterated over and over again, but that it is also a cumulative, potentially autocatalytic process. This suggests

Hypothesis 5 By re-using newly created concepts in further iterations of the generative operation, an infinitely growing number of concepts can emerge from a finite number of initial elements, provided that the share of combinations to which a new meaning can be attributed is non-vanishing.

In the light of Hypothesis 5, the evolution of human thought appears to be the creation of increasingly more complex chains of concepts composed of an ever larger set of known elements. (What carries novelty are, in each case, the recombined elements that did not previously exit.) This fact is hidden only by the complexity-reducing habit of denoting longer chains, or parts of them, with own shorthand terms. For example, instead of using the combinatory extension (ii-c) "gas stations selling gas and convenience goods", a shorthand has already emerged, calling the new hybrid a "supercenter". In this example, Hypothesis 5 would thus presuppose that "supercenter" can be entered into the formation of new propositional networks, perhaps as subject or object, without any of the now three concepts "kiosks", "gas stations", and "supercenter" being a substitute for any other.

If Hypothesis 5 is correct, it follows by backward induction that at the beginning of the chain of cumulative novelty creation, there must have been some ultimate prior concepts that were themselves not cognitively created. To put it differently, the cumulative process must have started from some innate concepts, a conclusion that accords with the "embodied knowledge" hypothesis (cf. Hayek 1952 for an early discussion).

The conjecture of an autocatalytic expansion of the number of concepts in human thought (i.e. of an expansion that, driven by a growth-of-base effect, fuels itself) is in agreement with the 
$-17-$

observable accumulation of the human knowledge. At the symbolic level of the alphabet, the noveltyby-extension phenomenon finds its equivalent in the ever-longer strings of letters by which the expanding body of thought (the "library of human thought" that has been growing since the invention of writing) is documented. However, given that it is not known what exactly happens in the interpretative operation, it is hard to assess what chances there are for the condition to hold that the share of combinations to which a new meaning can be attributed is indeed non-vanishing. Furthermore, it cannot be excluded that, because of both memory and communication constraints, it is increasingly more difficult to make the ever growing body of concepts available for further (re-) combinations. $^{4}$

\section{Degrees of Novelty and Uncertainty}

Novelty has been defined here as something that was unknown before a particular point in time and that, hence, was discovered or created at that point in time. However, whether a particular novelty is only a more or less minor detail of something that is otherwise well known or whether it is altogether new seems to make a big difference. The invention of a new ignition device like the sparking plug, for instance, created a new and more reliable way of running a combustion engine, but in retrospect, this seems to be a less significant novelty than the invention of the combustion engine itself. Observations such as these give rise to the conjecture that there are degrees of novelty that can differ from case to case. In fact, such an ex post distinction between degrees of novelty is often made in innovation

4 For a discussion of the economic implications of an auto-catalytic growth of economically useful knowledge see Weitzman (1998). For scientific knowledge it has been claimed that there is evidence for a fading auto-catalytic growth of knowledge primarily because there is a declining share of significant new results from search and experimentation; see de Solla Price (1963) and Rescher (1989). 
research, for example, by contrasting basic innovations with follow-up innovations. ${ }^{5}$ The question is whether different degrees of novelty can also be distinguished ex ante. Such differences correlate with the uncertainty surrounding an upcoming novelty. The higher the degree of novelty, the more noveltyinduced uncertainty seems to loom. To determine the extent of novelty-induced uncertainty, it would therefore be desirable to have a measure for the ex ante degree of novelty.

In a procedural approach to novelty it is straightforward to try to derive differences in the degree of novelty from constraints that can be imposed on the generative and/or interpretative operation on a priori grounds. An example of such constraints was given above with the contextual constraints relevant for the combinatory extensions (i-c) and (ii-c). Indeed, if the generative operation and its outcomes are known, those combinations that cannot be generated by that operation can be excluded from the subsequent interpretative operation, thus reducing substantially the input set on which it is based.

A simple example is an urn with 49 balls numbered from 1 to 49 and a mechanical device by which 6 balls are drawn from that urn at random in an unbiased way without replacement and without observing the order of the drawings. (This is the basis of a popular weekly bet offered by the state lottery in some European countries.) In this example, no outcome other than a combination of six different integers between 1 and 49 can ever be generated. To give another example, imagine a unit box that is subjected to the "baker transformation" (Prigogine 1993). This means that the box is squashed to half its height and double its length, cut in the middle, and re-composed by putting the

5 See the examples in Silverberg and Verspagen (2003). The distinction can be traced back to Schumpeter (1934, pp. 64-66) who stresses in his theory of entrepreneurial innovations the role of novelty that discontinues previous lines of technological and organizational development. The deeper issue here are the gradualist vs. saltationalist theories of evolution (see Rosser 1992 for a discussion) that implicitly presuppose a distinction of different degrees of novelty. 
$-19-$

cut-off right half on top of the left. The outcome of the transformation is the spatial relocation of the coordinates of all points in the unit box by a deterministic rule, yet the future coordinates of any two initially chosen points disappear quickly from sight, the further ahead an iteration of the transformation is. All that can (trivially) be excluded in this example is that no point will ever fall outside the unit box.

Regarding the interpretative operation, the fact that it is not fully understood makes it difficult, if not impossible, to impose ex ante constraints on it or its outcome. What can often be observed, but should not be confused with the desirable constraints, is that criteria are invoked ex ante by which the outcome of the interpretative operation is to be assessed ex post. For example, for a given technical or economic problem, some desirable features of its unknown solution may be specified in advance. The novelty resulting from the subsequent creative search process may then be assessed ex post as to whether it represents a solution to the problem with the desired features.

The deviation of actually emerging novelty from the expectations formed before may ex post be taken as a measure of subjective surprise. It is clear, though, that such an ex post assessment of surprise cannot have any bearing on an ex ante degree of novelty, nor can an ex ante assessment of "potential surprise" have any bearing. The cardinal measure of potential surprise was suggested by Shackle (1949) as an alternative for deriving subjective probabilities. It rates events that are ex ante imagined (and hence anticipated) by a decision maker according to how surprising their later occurrence would subjectively be felt by the decision maker. In contrast, novelty is something that, by definition, is not anticipated. ${ }^{6}$

6 A striking example is the "Arcanum" story (Gleeson 1999). In 1703 the German alchemist Johann Friedrich Boettger was imprisoned by August the Strong of Saxonia, because it was believed that he would be able to synthesize gold. A laboratory was set up for him at the fortress in Meissen. He probed and sifted there using several processes and materials, but instead of finding a recipe for 
It seems, thus, that the interpretative operation is either non-trivial, so that no constraint can ex ante be imposed on the operation and its outcomes, ${ }^{7}$ or it is a trivial operation in the sense that the meaning of all possible outcomes is already known beforehand. This is, in particular, the case when the generative operation implies a binding interpretation for all its outcomes as in the examples of the (stochastic) lottery and the (deterministic) baker transformation. No associative act is needed anymore for making sense of the results. In view of this condition, the conclusion that can be derived from the feasible ex ante constraints is rather weak.

Hypothesis 6 The ex ante degree of novelty is minimal (but not necessarily zero) if the generative operation is known and the interpretative operation is trivial in the sense that the meaning of all its possible outcomes is known beforehand. The degree of novelty is maximal where the generative operation is not known and no constraint can be imposed on the outcome of the interpretative operation.

Turning to the relationship between the degree of novelty and uncertainty, two questions can

making gold, six years later he invented a way to make white porcelain. A non-anticipated outcome like this (it is actually a case of a domain-specific novelty as, unknown to the western world, the way to make white porcelain had been known in China since ancient times) cannot be made part of an ex ante rating of potential surprise.

7 Note that even an apparently trivial constraint like that of logical truth is not binding here. The construction of an erroneous mathematical proof may be as much novelty as the construction of a logically true one. The same holds for the validity of an empirical conjecture created by inductive inference; it is new independent of whether or not it can be confirmed. This is indeed the very basis for running science as a process of conjectures and refutations. 


\section{$-21-$}

be posed. First, while the limiting case of a maximal degree of novelty is obviously associated with a state of complete (novelty-induced) uncertainty about what is going to be created, what is the nature of novelty-induced uncertainty in the other limiting case? Second, and following-up an answer to this question, what can be said about the degree of novelty, or novelty-induced uncertainty, in the range between the two extremes?

In the case of a minimal degree of novelty, to start with that question, there is still something not known ex ante. Consider the lottery example. Assuming that the true bias status of the drawing device is known, all possible outcomes of a future run can be anticipated. However, because it is generated by a random mechanism, what is not yet known is the particular subset of integers drawn at a certain date or iteration in the future. However, precisely because all possible outcomes and the nature of the sampling mechanism are known, there is a basis for deriving probabilities. Lacking evidence to the contrary, all possible outcomes can be treated as equally probable so that degrees of likelihood of, or belief in, all possible outcomes can be constructed as a cardinal measure of uncertainty in the probabilistic sense.

More generally speaking, the feature that is still unknown in a state where the degree of novelty is minimal is exactly the object on which probability theory focuses when arguing that something is not yet known with certainty. An instance indicative of this constellation is Zabell's (1992) attempt at "predicting the unpredictable". Zabell explores the emergence of novelty under the assumption that the emergence is a serial phenomenon. His example is the emergence of a new species. A finite number of organisms already exist. They are classified into different species. New organisms are continually added. They can either represent an already known species or a new one (the so-called "sampling of species problem"). Zabell is able to derive the probability for the event that a newly added organism represents a new species. In terms of the present approach, what Zabell predicts has a minimal degree of novelty. As in the case of the lottery, all possible outcomes of the generative operation can be anticipated. The interpretative operation is trivial, adding no new meaning to any of the two possible outcomes. What is not known with certainty is whether or not the next 
living organism added will indeed increase the number of existing species by 1 .

Regarding the second question above, it can be more specifically asked now whether the reduction of novelty-induced uncertainty to uncertainty in the probabilistic sense is also possible for a degree of novelty in the range between minimal and the maximal ones. By Hypothesis 6, the degree of novelty is greater than minimal, but less than maximal, if one of the following conditions holds. Either the outcome of the interpretative operation is non-trivial, but possible outcomes of the generative operation are known ex ante, or the outcome of the interpretative operation is trivial, but the outcome of the generative operation cannot be anticipated.

Consider the first condition. Since the meaning of what comes out of the interpretative operation is not known beforehand, the information available ex ante is no longer sufficient to specify the state space required for constructing a probability measure. Zabell's "sampling of species problem" can be used to illustrate the difference. The outcome of the interpretative operation would not be trivial (and, hence, the ex ante degree of novelty is greater than minimal) if, instead of predicting the mere occurrence of a new species, the problem were to predict the properties of the new species. However, in contrast to Zabell's probability model in which the state space can recursively be determined by adding a further organism of either an already existing species or a new one, the state space would now have to be made dependent on the number of different properties of the new species, something not yet known. ${ }^{8}$

8 When such information is lacking, probability theory traditionally resorts to the notion of equi-probability or equi-possibility to compensate for the information deficit (see Hacking 1975, Chap. 14). However, these notions cannot be extended to an as yet unknown number of cases or variants, except in the trivial sense that everything is possible, see Shackle (1973, Chap.1) and his "residual hypothesis" verdict. 


\section{$-23-$}

Now consider the case where the interpretative operation is trivial, but the outcome of the generative operation cannot be anticipated. Again, there is no basis for specifying the state space required for a probability model. An instructive example is the process resulting from Prigogine's iterated baker transformation. Whatever the transformation generates, its interpretation is indeed known beforehand: it will always be the coordinates of two points in the unit box. In fact, even the generative operation is clearly defined by the deterministic relocation rule. Nonetheless, in many cases, the position which two initially chosen points subsequently take can no longer be anticipated for iterations further ahead into the future. ${ }^{9}$ The outcome needs to be induced by carrying out the

9 It is sufficient here to demonstrate this for the horizontal distance $\mathrm{d}, 0<\mathrm{d} \leq 1$, between the two points (cp. Prigogine 1993). $\mathrm{d}$ is multiplied by a "factor 2 modulo 1 " transforming $\mathrm{d}$ into

$$
\mathrm{d}^{\prime}= \begin{cases}2 \mathrm{~d}, & \text { if } 0<2 \mathrm{~d} \leq 1 \\ 2 \mathrm{~d}-1, & \text { if } 2 \mathrm{~d}>1 .\end{cases}
$$

Any value that $d$ can take can be represented as the sum of some elements of the geometric series $2^{-\mathrm{n}}$, $\mathrm{n}=0,1,2, \ldots$ Mark the position of each of the elements in the geometric series that sum up to $\mathrm{d}$ with 1 and all other positions up to the smallest element of the sum with 0 . This results in a binary string of varying length. Each iteration of the baker transformation can then be expressed in terms of a Bernoulli shift (i.e. a shift by which the zeros and ones of the binary string are moved by one position)

to the left. Now let $d=\sqrt{ } 2-1$. In this case, the number of elements in the geometric series required to represent this value, and hence the corresponding binary string, are infinite. It is not possible, therefore, to derive the evolution of $d$ by extrapolation of the Bernoulli shift. This is a simple case of a chaotic time series in which novelty can emerge (cp. also Smale's 1967 "horseshoe" model). 
generative operation over and over again.

For the relationships between the concept of novelty-induced uncertainty on the one hand and the probabilistic concept of uncertainty on the other, it follows from these considerations:

Hypothesis 7 Novelty-induced uncertainty reduces to uncertainty in the probabilistic sense measured by the degree of likelihood or belief if, and only if, the degree of novelty is minimal. In the limiting case of a degree of novelty equal to zero, known outcomes occur with probability 1 (certainty).

On a priori grounds our knowledge does not suffice to weigh how lacking information about the generative operation impacts the degree of novelty compared to the impact of a lack of information about the interpretative operation. A comparative assessment of the number, relevance, and/or conditionality of possible constraints on the two operations is not feasible. For this reasons there is no possibility of deriving a consistent ranking for the ex ante degree of novelty between the minimal and the maximal degrees, not to speak of a cardinal measure. Consequently, what our knowledge allows is only the weakest possible ordering (cp. Keynes 1921, Chap. II).

Hypothesis 8 The ex ante degree of novelty (and, hence, novelty-induced uncertainty) can, on a priori grounds, be determined as being maximal, less than maximal but greater than minimal, or minimal (with limiting case of a zero degree of novelty).

Because of its peculiar epistemological status, novelty-induced uncertainty is a much less structured concept than uncertainty in the probabilistic sense. ${ }^{10}$

10 If a distinction between risk and uncertainty as in Knight (1921, Chap. VII) is made, noveltyinduced uncertainty is a case of Knightian uncertainty. 


\section{$-25-$}

\section{Conclusions}

Why is it so difficult to work with the notion of novelty? How does novelty emerge? Are there different degrees of novelty, and if so, how do they relate to the concepts of uncertainty? In this paper an attempt has been made to answer these questions. Novelty was defined as something that was unknown before a particular point in time and that, hence, was discovered or created at that point in time. It was argued that novelty emerges from two logically distinct operations, a generative one and an interpretative one. To take advantage of our capacity to grasp the meaning of novelty intuitively, a semantic representation of novelty was chosen to explain this in more detail. The generative operation was shown to (re-) combine conceptual inputs given here in the form of propositional networks. With respect to the interpretative operation, it had to be acknowledged that the way in which meaning (if any) is attributed in a conceptual integration to the newly generated combinations is not yet understood and that it therefore cannot be carried out outside the human mind. Nonetheless, the insights into the generative and the interpretative operations that are feasible allow some conclusion to be drawn.

One of the conclusions is that the peculiar epistemic status of novelty is caused by the inductive nature of the operations from which it emerges. These operations need to be carried out in real time before any novelty can be revealed. Another conclusion is that, by the very operation by which they are generated, all newly created cognitive concepts are made up of elements that have previously been known. Since newly created concepts can be used, in turn, as inputs for iterating the generative operation, ever more extended compositions of known elements evolve. Starting from conceptual input sets with a finite number of elements, an infinite number of new concepts can be created. The expansion of human thought is thus a cumulative process that feeds itself.

Since it seems to make a big difference whether a particular novelty is only a minor variation of something already known or whether it is altogether new, the paper also discussed the possibility of 


\section{$-26-$}

distinguishing between different degrees of novelty ex ante. Differences in this degree correlate with the uncertainty surrounding an upcoming novelty. In the procedural approach to novelty that was chosen in this paper, differences in the degree of novelty are likely to result from constraints that can be imposed on the generative and/or interpretative operations on a priori grounds. Such constraints were shown to relate to how much is known about the properties of the two operations and their possible outcomes.

Accordingly, the degree of novelty and, hence, the uncertainty surrounding an up-coming novelty were argued to be maximal where the generative operation is not known and no constraint can be imposed on the outcomes of the interpretative operation. It could be demonstrated that, at the other end of the range, the ex ante degree of novelty is minimal, but not necessarily zero, if the outcomes of the generative operation and the meaning of all possible outcomes of the interpretative operation are known beforehand. There may then still be features of up-coming novelty that are not known with certainty, but for these it is possible to derive degrees of likelihood. Hence, novelty-induced uncertainty in this special case reduces to uncertainty in the probabilistic sense. For degrees of novelty between the two extremes, novelty-induced uncertainty cannot be reduced to uncertainty in the probabilistic sense, nor can, for epistemological reasons, an ordinal or even cardinal measure of the degree of novelty be established.

\section{References}

Anderson, J.R., 2000. Cognitive Psychology and Its Implications. $5^{\text {th }}$ edition. New York: Worth Publishers.

Bäck, T., 1996. Evolutionary Algorithms in Theory and Practice. Oxford: Oxford University Press. Boden, M.A., 1999. Computer models of creativity. In: Sternberg, R. J. (Ed.). Handbook of Creativity. Cambridge: Cambridge University Press, 351-372.

Campbell, D.T., 1960. Blind variation and selective retention in creative thought as in other 
knowledge processes. Psychological Review 67, 380-400.

de Solla Price, D.J, 1963. Little Science, Big Science. New York: Columbia University Press.

Edelman, G.M., Tononi, G., 2000. A Universe of Consciousness: How Matter Becomes Imagination. New York: Basic Books.

Fauconnier, G., Turner, M., 2002. The Way We Think - Conceptual Blending and the Mind's Hidden Complexities. New York: Basic Books.

Finke, R.A., Ward, T.B., Smith, S.M., 1992. Creative Cognition - Theory, Research, and Applications. Cambridge, MA: MIT Press.

Gentner, D., Markman, A.B., 1997. Structure mapping in analogy and similarity. American Psychologist 52, 45-56.

Gleeson, J., 1999. Arcanum. New York: Warner Books.

Hacking, I., 1975. The Emergence of Probability. Cambridge: Cambridge University Press.

Hayek, F.A., 1952. The Sensory Order - An Inquiry into the Foundations of Theoretical Psychology. London: Routledge and Kegan Paul.

Hodgson, G.M., Knudsen, T., 2006. Why we need a generalized Darwinism, and why generalized Darwinism is not enough. Journal of Economic Behavior and Organization 61, 1-19.

Hofstadter, D.R. and the Fluid Analogies Research Group 1995. Fluid Concepts and Creative Analogies. New York: Basic Books.

Holland, J.H., 1992. Adaptation in Natural and Artificial Systems. $2^{\text {nd }}$ edition. Cambridge, MA: MIT Press.

Johnson-Laird, P.N., 2005. Flying bicycles: How the Wright Brothers invented the airplane. Mind \& Society 4, 27-48.

Keynes, J.M., 1921. A Treatise on Probability. London: MacMillan.

Knight, F.H., 1921. Risk, Uncertainty and Profit. Chicago: Chicago University Press.

Koch, C., Crick, F., 2000. Some thoughts on consciousness and neuroscience. In: Gazzaniga, M.S. (Ed.). The New Cognitive Neurosciences. $2^{\text {nd }}$ edition. Cambridge, Mass.: MIT Press, 1285-1294. Koestler, A., 1964. The Act of Creation. London: Hutchinson \& Co. 
Kohonen, T., 1987. Self-organization and Associative Memory. $2^{\text {nd }}$ edition. Berlin: Springer.

Machlup, F., 1980. Knowledge and Knowledge Production. Princeton: Princeton University Press.

Markose, S.M., 2004. Novelty in complex adaptive systems (CAS) dynamics: A computational theory of actor innovation. Physica A: Statistical Mechanics and Its Applications 344, 41-49.

Markose, S.M., 2005. Computability and evolutionary complexity: Markets as complex adaptative systems. Economic Journal 115, F159-F192.

Mokyr, J., 1990. Twenty-Five Centuries of Technological Change - An Historical Survey. Chur (Switzerland): Harwood.

Nersessian, N.J., 2002. Abstraction via generic modeling in concept formation in science. Mind \& Society $3,129-154$.

Newell, A., Simon, H.A., 1972. Human Problem Solving. Englewood Cliffs: Prentice Hall.

Prigogine, I., 1993. Bounded rationality: From dynamical systems to socio-economic models. In: Day,

R. H., Chen, P. (Eds.). Nonlinear Dynamics and Evolutionary Economics. Oxford: Oxford University Press, 3 - 13 .

Rescher, N., 1989. Cognitive Economy: The Economic Dimension of the Theory of Knowledge. Pittsburgh: University of Pittsburgh Press.

Rogers, E., 1995. Diffusion of Innovations. New York: Free Press.

Rosser, J.B. Jr,. 1992. The dialogue between the economic and the ecologic theories of evolution. Journal of Economic Behavior and Organization 17, 195-215.

Schumpeter, J.A., 1934. Theory of Economic Development. Cambridge, MA: Harvard University Press.

Shackle, G.L.S., 1949. Expectation in Economics. Cambridge: Cambridge University Press.

Shackle, G.L.S., 1973. Epistemics and Economics - A Critique of Economic Doctrines. Cambridge: Cambridge University Press.

Silverberg, G., Verspagen, B, 2003. Breaking the waves: A Poisson regression approach to Schumpeterian clustering of basic innovations. Cambridge Journal of Economics 27, 671-693.

Smale, S., 1967. Differentiable dynamical systems. Bulletin of the American Mathematical Society 
$73,747-817$.

Sternberg, R.J. (Ed.), 1999. Handbook of Creativity. Cambridge: Cambridge University Press.

Swift, J., 1934. Gulliver's Travels and Selected Writings in Prose and Verse. Hayward, J. (Ed.). New York: Random House.

Usher, A.P., 1954. A History of Mechanical Inventions. Cambridge, Mass.: Harvard University Press. Vincenti, W.G., 1990. What Engineers Know and How They Know It. Baltimore: Johns Hopkins University Press.

Weitzman, M.L., 1998. Recombinant growth. The Quarterly Journal of Economics 113, 331-360.

Witt, U., 1993. Emergence and dissemination of innovations: Some principles of evolutionary economics. In: Day, R. H., Chen, P. (Eds.). Nonlinear Dynamics and Evolutionary Economics. Oxford: Oxford University Press, 91-100.

Zabell, S.L., 1992. Predicting the unpredictable. Synthése 90, 205-232. 